Article

\title{
Research on the Real-Time Distributed Economic Dispatch Strategy for Microgrids
}

\author{
Jian Le *, Qian Zhou, Liangang Zhao and Yang Wang \\ School of Electrical Engineering and Automation, Wuhan University, Wuhan 430072, China; \\ zlsy2014@whu.edu.cn (Q.Z.); zzf20141201@163.com (L.Z.); whugoodz@163.com (Y.W.) \\ * Correspondence: lej01@tsinghua.org.cn
}

Received: 27 August 2019; Accepted: 18 October 2019; Published: 21 October 2019

\begin{abstract}
A microgrid (MG) is one of the most efficient ways to cope with the grid-connection of a large number of small-sized distributed energy resources. This paper presents a consensus-based fully distributed economic dispatch (ED) strategy for MGs, with the aim of tackling the difficulties of existing algorithms in modeling network power loss and providing global information. The external power grid to which the MG connects is treated as a special power source called a virtual generator, and participates in the economic dispatch process. Taking the incremental cost of a power generator as the consensus variable, a distributed ED model was formulated based on consensus protocol and a sub-gradient-based optimization method for solving this model has been proposed. The convergence of the distributed ED system was investigated by utilizing matrix spectrum radius analysis theory. The effectiveness of the proposed strategy was verified by carrying out simulation under normal operation of the MG, both with and without the consideration of network power loss. Moreover, simulation results under several scenarios, including exchanged power order variation and distributed generation plug and play, are provided to demonstrate the robustness of the distributed ED strategy.
\end{abstract}

Keywords: consensus protocol; distributed economic dispatch; virtual power generator; distributed sub-gradient optimization; microgrid

\section{Introduction}

Climate change and environmental concerns stimulate the development of the concept of distributed energy resources (DERs) and their subsequent widespread application in power systems across the world [1]. DERs have the advantages of economy, efficiency and reliability; however, the integration of a large number of small- and ultra-small-sized, geographically dispersed and heterogeneous DERs has a greater impact on the power grid and presents austere challenges to the network operator in controlling and managing the whole system [2]. One of the proposed solutions is the concept of a microgrid (MG), driven by recent advances in both modern communication and intelligent computation technologies [3,4].

Economic dispatching (ED) is also one of the most fundamental issues of an MG, where the total required generation is distributed among the generators in operation by minimizing the selected cost criterion, subject to load demand and generator capacity constraints [5]. Traditionally, solution algorithms for the ED problem of MGs have been centralized in nature, wherein a powerful central computer is employed to make a decision on the power commitment for each power generation unit by solving a specific form of optimization problem, according to the information collected via communication links deployed between the central computer and each generation unit [6]. As pointed out in [7], the design of intelligent economic dispatching strategies for microgrids is drastically different to that of conventional central grids due to two unique challenges. Firstly, the demand and renewable generation uncertainty emphasizes the need for online algorithms. Secondly, the widely adopted peak-based pricing 
scheme results in the need for new peak-aware strategy design. Therefore, artificial-intelligence-based approaches such as particle swarm and simulated annealing algorithms have also been applied in order to optimally allocate the power of different types of generators and energy storage units [8,9]. These approaches are still unable to tackle the difficulties inherent to the solving of the ED problem in a centralized framework. These problems include the fact that the dispatching center is usually associated with a heavy computation and communication burden, the overall dispatching system is very fragile with one-point failure of the central computer, and more importantly, that the whole system needs to be reconstructed for the adding and/or leaving of a power generator [10].

The transition of power generation and distribution in a power grid to become increasingly decentralized stimulates the development of distributed control systems that are efficient in handling dynamic loads, robust against transmission and generation failure and allow for plug-and-play of DERs [11,12]. The majority of them rely on consistency or distributed optimization algorithms that have been extensively studied and applied in various engineering fields, such as distributed sensor networks [13], unmanned aerial vehicle formation [14] and robot cooperative control [15].

Consequently, there has been considerable interest in developing distributed strategies for the solution to the ED problem of MGs [16,17]. The predominant approach is to consider quadratic cost functions for generators and perform consensus over their incremental costs under local communication topologies [18]. The basic idea and implementation method of a leader-follower type distributed ED algorithm has been presented in $[19,20]$. The leader performed the so-called pinning control according to the power mismatch of the system and the optimal power allocation was obtained when the incremental costs of all the units were equal. However, the power mismatch between total load and total power generation is global information that is very difficult to obtain by the leader locally in a distributed framework. Although the knowledge of power mismatch was no longer needed, the distributed ED algorithm proposed in [21] assumed that the leader had access to the information on the total load demand, which is, however, also a type of global information. Alternatively, in a leaderless version of the distributed ED strategy [22], each power generation unit adjusted its output based on the feedback on the power mismatch estimated locally by the generation unit itself. This algorithm should be initialized with a feasible power allocation. In [23], the authors proposed a finite-time convergence distributed ED strategy with the aim to speed-up the iteration process, but its implementation required placing an agent on each bus integrated with a load or a power generator. Network transmission power loss is also an important factor affecting the ED solution. Transmission power loss was handled in the strategy proposed in [24] on the premise that each generator can estimate the power loss in the transmission lines adjacent to it. In a centralized framework, power loss can be roughly estimated or accurately calculated based on power flow analysis by the dispatching center. However, it is scarcely possible for a power generation unit itself to accurately estimate power loss that is time-variant, network operation-dependent and distributed all over the network, even with the help of a sophisticated distributed algorithm.

This paper presents a novel leader-follower type distributed ED planning for an MG, to tackle the difficulties of existing distributed ED algorithms in modeling network power loss and providing global information. The major innovations of this paper are as follows: (1) We developed a novel leader-follower type distributed ED framework which treats the external power grid as a special power unit called a 'virtual power generator', that is 'dispatched' by the MG operator. This particular design greatly facilitates the synthesis of our distributed information exchange protocol in a unified manner, and the local information of power mismatching and exchanged power instruction can be shared with other power units in accordance with the rule of the consensus protocol. The participation of the virtual power generator has no effect on the economic dispatching solution at all. (2) A fully distributed leader-follower ED model has been established. It only requires the leader to access the information of power mismatching and exchanged power instruction locally, thus avoiding the complexity in obtaining global information. In addition, network power loss can also be treated conveniently and modeled simply in the model formulation. To our best knowledge, this model is the 
first full version of a quadratic cost function-type distributed ED model. (3) We developed a distributed sub-gradient-based method in order to solve the built ED model, and to provide the strict proof to investigate the convergence of this method for the first time.

The remainder of this paper is structured as follows: in Section 2, the framework of our distributed economic dispatch system based on the concept of a virtual power generator is described. The real-time distributed economic dispatch model and its subgradient-based optimal solution are presented in Section 3. Section 4 investigates the convergence of the distributed economic dispatch system. Simulation results under several scenarios are provided in Section 5 to show the effectiveness of the proposed strategy. Finally, in Section 6, the main findings of the paper are summarized.

\section{Distributed ED Framework of an MG}

Figure 1 shows the general structure of an MG. It is normally integrated with several fossil fuel-based generators, gas turbo-generators and heterogeneous distributed renewable resources, such as photovoltaics (PV), wind-turbine (WT) power and small-scale hydro-turbine generators. The goal of economic dispatch is to minimize the total power generation cost in scheduling all the power sources to balance the load demand in the MG and to satisfy the requirements of the power exchange between the MG and the external power grid. The instruction for the exchange of power may be issued directly from a system operator in a higher level of the hierarchical control system or the commitment can be made by the MG locally when participating in electricity market trading.

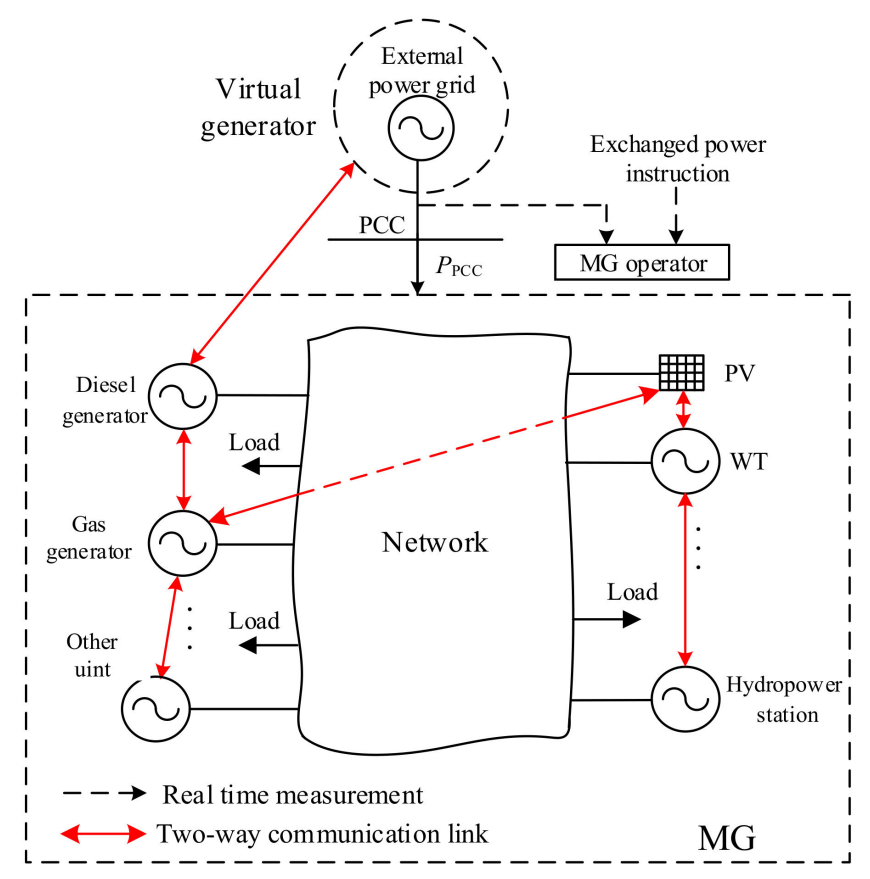

Figure 1. General structure of a microgrid (MG) with a virtual generator.

In a distributed economic dispatch scheme, the central scheduler is replaced by a collection of the local controllers of each generation unit, and the communication network between local controllers can be of arbitrary topology, as long as the topological graph is connected. The solution to a distributed ED problem involves an iteration process. In an iteration circle, each generation unit makes a decision regarding its power generation autonomously, by synthesizing the status of itself and the information of its neighbors, which is received via two-way communication links (the red arrowhead lines shown in Figure 1). The economic dispatch goal of the MG can be achieved once the iteration computation process reaches convergence. 
To completely tackle the difficulty of existing distributed ED algorithms in collecting global information and effectively consider the power exchanging requirement in the dispatch process, we introduced a novel scheme wherein the external grid to which the MG connects is regarded as a special power generation unit called a 'virtual power generator'. Here, 'virtual' means that this generator is in fact a non-schedulable unit and just participates in the iteration computation process. This is in order to provide the real-time information on the mismatch between the exchanged power $P_{\text {PCC }}$ and its instruction $P_{\text {ref }}$, in a way conforming to the rule of the consensus-based protocol that only consensus variables can be transmitted. In this sense, this virtual power generator is always 'controlled and dispatched' by the MG operator due to fact that the operator can measure the exchanged power $P_{\mathrm{PCC}}$ more conveniently and is the specific person to receive or generate the instruction of the exchanged power.

\section{Distributed ED Model and Optimization Solution}

\subsection{Distributed Economic Dispatch Model}

A regular centralized ED model of an MG can be formulated as:

$$
\begin{aligned}
& \min F=\sum_{i=1}^{n} F_{i}\left(P_{\mathrm{G}, i}\right) \\
& \text { s.t. }\left\{\begin{array}{l}
\sum_{i=1}^{n} P_{\mathrm{G}, i}+P_{\mathrm{ref}}=P_{\mathrm{D}}+P_{\mathrm{LOSS}} \\
P_{\mathrm{G}, i}^{\min } \leq P_{\mathrm{G}, i} \leq P_{\mathrm{G}, i}^{\max }, i=1,2, \ldots, n
\end{array}\right.
\end{aligned}
$$

where $n$ is the number of power source units participating in the economic dispatch; $P_{\mathrm{G}, i}$ and $F_{i}\left(P_{\mathrm{G}, i}\right)$ is the power and cost function of unit $i$, respectively; $P_{\mathrm{G}, i}^{\min }$ and $P_{\mathrm{G}, i}^{\max }$ is, respectively, the power lower and upper limit of unit $i ; P_{\mathrm{D}}$ is the total load demand of the MG, $P_{\text {LOSS }}$ is the network power loss and $P_{\text {ref }}$ is the order of the power exchanged between the MG and the external grid.

The cost function $F_{i}\left(P_{\mathrm{G}, i}\right)$ of the real power source unit $i$ is usually expressed as a quadratic function of its power $P_{\mathrm{G}, i}$, that is:

$$
F_{i}\left(P_{\mathrm{G}, i}\right)=c_{i}+b_{i} P_{\mathrm{G}, i}+a_{i} P_{\mathrm{G}, i}^{2}
$$

where $a_{i}, b_{i}$ and $c_{i}$ are the corresponding coefficients.

Using the classical Lagrange multiplier method, the optimal model (1) with an equality constraint can be converted into:

$$
\left\{\begin{array}{l}
\min F=\sum_{i=1}^{n} F_{i}\left(P_{\mathrm{G}, i}\right)+\lambda \Delta P \\
\text { s.t. } P_{\mathrm{G}, i}^{\min } \leq P_{\mathrm{G}, i} \leq P_{\mathrm{G}, i}^{\max }
\end{array}\right.
$$

where $\lambda$ is Lagrange multiplier and:

$$
\Delta P=P_{\mathrm{D}}+P_{\mathrm{LOSS}}-P_{\mathrm{ref}}-\sum_{i=1}^{n} P_{\mathrm{G}, i}
$$

For constant network power loss and convex cost-power functions, the optimal solution $P^{*} \mathrm{G}=$ $\left[P_{\mathrm{G}, 1}, P_{\mathrm{G}, 2}, \ldots, P_{\mathrm{G}, \mathrm{n}}\right]$ and $\lambda^{*}$ of Formula (3) is unique and can be characterized by:

$$
\left\{\begin{array}{l}
\frac{d F_{1}\left(P_{\mathrm{G}, 1}\right)}{d P_{\mathrm{G}, 1}}=\ldots=\frac{d F_{n}\left(P_{\mathrm{G}, n}\right)}{d P_{\mathrm{G}, n}}=\lambda^{*} \\
\sum_{i=1}^{n} P_{\mathrm{G}, i}=P_{\mathrm{D}}+P_{\mathrm{LOSS}}-P_{\text {ref }}
\end{array}\right.
$$


Derivation $d F_{i}\left(P_{\mathrm{G}, i}\right) / P_{\mathrm{G}, i}$ is also termed as the incremental cost of unit $i$. The ED model (3) can be solved conveniently in a centralized mode, provided that all the cost functions $F_{i}\left(P_{\mathrm{G}, i}\right)$, total load demand $P_{\mathrm{D}}$, power loss $P_{\text {LOSS }}$ and power order $P_{\text {ref }}$ are foreknown by the central scheduler.

We introduced a virtual generator with the sequence number ' 1 ' to decompose the centralized ED mode (1). The local ED model of each real generator and the virtual generator can be uniformly formulated as:

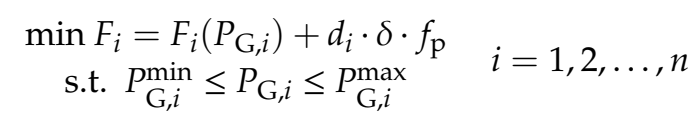

where $d_{i}$ is used to identify the virtual power generator, i.e., $d_{1}=1$ and $d_{\mathrm{i}}=0$ for $i=2,3, \ldots, n$ in this paper.

We simply set the cost function $F_{1}\left(P_{\mathrm{G}, 1}\right)=0$ and the power limit $P_{\mathrm{G}, 1}^{\min }=-\infty$ and $P_{\mathrm{G}, 1}^{\max }=+\infty$ for the virtual power generator. According to the introduction of the virtual generator in Section 2, we can know that its power $P_{\mathrm{G}, 1}$ equals the power exchanged between $\mathrm{MG}$ and the external grid, i.e., $P_{\mathrm{G}, 1}=P_{\mathrm{PCC}}$.

In accordance with the centralized ED model (1), function $f_{\mathrm{p}}$ in (6) is defined as follows:

$$
f_{\mathrm{p}}=\frac{1}{2}\left(P_{\mathrm{D}}+P_{\mathrm{LOSS}}-\sum_{i=1}^{n} P_{\mathrm{G}, i}-P_{\text {ref }}\right)^{2}
$$

In real operation, we can know that:

$$
P_{\mathrm{D}}+P_{\mathrm{LOSS}}-\sum_{i=1}^{n} P_{\mathrm{G}, i}=P_{\mathrm{PCC}}
$$

Thus, Formula (7) can be reformed as the following:

$$
f_{\mathrm{p}}=\frac{1}{2}\left(P_{\mathrm{PCC}}-P_{\text {ref }}\right)^{2}=\frac{1}{2}\left(P_{\mathrm{G}, 1}-P_{\text {ref }}\right)^{2}
$$

We can learn by investigating Formulas (6)-(9) that: (1) The sum of the local model (6) for $n$ power units (including the virtual power generator) collectively equals the central ED model (1); (2) Since $d_{\mathrm{i}}=0$ when $i=2,3, \ldots, n$, model (6) can be built by a real power source unit itself locally and independently. As for the virtual generator, it only needs the information on the real-time exchanged power $P_{\mathrm{PCC}}$ and the power order $P_{\text {ref }}$ that can be provided by the MG operator conveniently to calculate the function $f_{p}$ in its local model (6). Therefore, the ED model (6) for $n$ power units is fully distributed in nature. (3) Global information such as total load $P_{\mathrm{D}}$ and the sum of the power of all the generators $\sum_{i=1}^{n} P_{\mathrm{G}, i}$ or the power mismatch $P_{\mathrm{D}}-\sum_{i=1}^{n} P_{\mathrm{G}, i}$ that are bottlenecks of current distributed ED strategies are no longer needed in developing our ED model (6). Furthermore, global operation information regarding power loss $P_{\text {LOSS }}$ that is very difficult to be calculated or estimated accurately can now be handled simply and conveniently.

\subsection{Sub-Gradient-Based Optimization Method}

The $n$ power source units and the communication links between them can be collectively regarded as a multi-agent system. Their interaction topology is represented using an undirected weighting graph $G=(V, E, A)$ with the set of nodes $V=\{1, \ldots, n\}$, edges $E \subseteq V \times V$ and an adjacency matrix $A$.

We took the incremental cost of a power generator as the consensus variable in order to develop the solution method for our distributed ED model (6). The discrete form of the protocol used by power generator $i$ to update its status in the $k$-th iteration can be formulated as the following: 


$$
\lambda_{i}[k+1]=\sum_{j=1}^{n} a_{i j} \lambda_{j}[k]+d_{i} \cdot \delta \cdot \Delta f_{\mathrm{P}}[k]
$$

where $k$ is the iteration number and $\lambda_{i}$ is the incremental cost of unit $i ; \delta$ is the step-size; coefficient $a_{i j}$, which is the weighting assigned by unit $i$ for the information received from unit $j$. In equal weighting scheme, $a_{i j}$ is chosen as follows:

$$
a_{i, j}= \begin{cases}1-\varepsilon \cdot\left|N_{i}\right|, & i=j \\ \varepsilon, & i \neq j \text { and } j \in N_{i} \\ 0, & i \neq j \text { and } j \notin N_{i}\end{cases}
$$

where $N_{i}=\{j \in V:(i, j) \in E\}$ is the set of neighbors of agent $i$, and edge $(i, j) \in E$ if agent $i$ can communicate with agent $j ;\left|N_{i}\right|$ denotes the number of neighbors of agent $i ; \varepsilon \in\left(0, d_{\max }\right)$ and $d_{\max }$ is the maximum degree of graph $G$.

Positive step-size $\delta$ plays an important role in determining the performance of a distributed algorithm in terms of convergence speed and optimization accuracy. Although it is possible to drive the errors between the decision variables and their optimal values to zero using, e.g., a diminishing step size rule [25], we prefer a constant step-size rule in view of its simplicity and relatively fewer number of iterations.

Based on the principle of sub-gradient distributed optimization method, if we tune $\Delta f_{\mathrm{P}}[k]$ as the negative partial differential of $f_{\mathrm{P}}$ at $\lambda_{0}[k]$ in the $k$ th iteration cycle, and we can obtain the following:

$$
\Delta f_{\mathrm{p}}[k]=P_{\mathrm{PCC}}[k]-P_{\text {ref }}
$$

The power order of the $i$-th $(i=1,2, \ldots, n)$ real power source unit after the $k$-th iteration is determined as follows:

$$
P_{\mathrm{G}, i}[k+1]=\frac{\lambda_{i}[k+1]-b_{i}}{2 a_{i}}
$$

Considering the power limits, the final power order of unit $i$ is the following:

$$
P_{G i}[k+1]=\left\{\begin{array}{l}
P_{G i}^{\min } \quad \frac{\lambda_{i}[k+1]-b_{i}}{2 a_{i}}<P_{G i}^{\min } \\
\frac{\lambda_{i}[k+1]-b_{i}}{2 a_{i}} \quad P_{G i}^{\min } \leq \frac{\lambda_{i}-b_{i}}{2 a_{i}} \leq P_{G i}^{\max } \\
P_{G i}^{\max } \quad \frac{\lambda_{i}[k+1]-b_{i}}{2 a_{i}}>P_{G i}^{\max }
\end{array}\right.
$$

Figure 2 shows the flow chart of our distributed ED strategy.

We can learn from the definition of $d_{i}$ that $\Delta f_{\mathrm{P}}$ is computed locally only by the virtual generator according to the measured exchanged power $P_{\mathrm{PCC}}$ and the instruction $P_{\text {ref }}$. More importantly, the introduction of the virtual generator is very helpful in formulating the distributed protocol (10) to share the information of $P_{\mathrm{PCC}}$ and $P_{\text {ref }}$ with all the other participants in accordance with the requirement of a distributed system that only the consensus variable can be transmitted. 


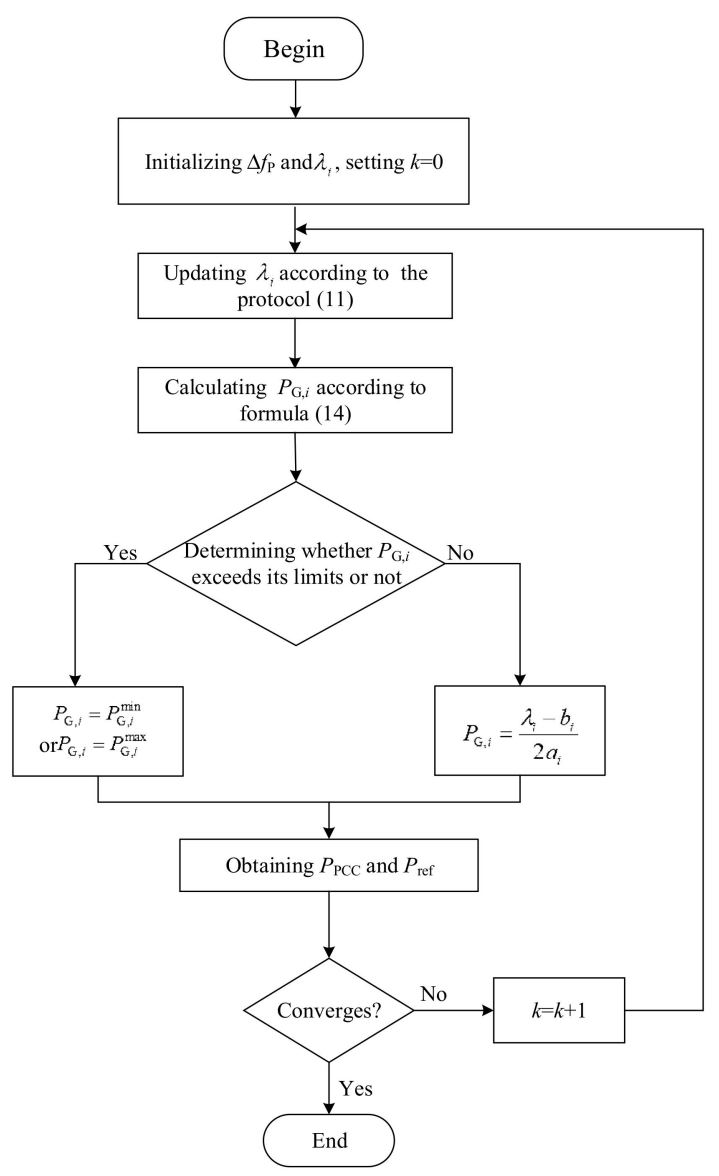

Figure 2. Flow chat of the real-time distributed economic dispatch (ED) strategy.

\section{Convergence Analysis of the Distributed ED System}

From Formula (12), we can know that $\Delta f_{\mathrm{P}}[k]$ is indeed the negative sub-gradient of function $f_{\mathrm{P}}$ (9) at $\lambda_{0}[k]$. Thus, our protocol (10) can be regarded as a special version of that proposed in [26] (see Formula (3) therein). Reference [26] has proven that the distributed computing method can obtain the same optimal solution as that derived by the centralized Lagrange multiplier method. The optimal solution can be solved from Formula (5) as follows:

$$
\lambda^{*}=\frac{P_{D}+P_{\text {LOSS }}-P_{\text {ref }}+\sum_{i=1}^{n} \frac{b_{i}}{2 a_{i}}}{\sum_{i=1}^{n} \frac{1}{2 a_{i}}}
$$

Let $\lambda^{*}$ to be the control target of the ED system, Formula (10) can be reformed as follows:

$$
\lambda_{i}[k+1]=\sum_{j=1}^{n} a_{i j} \lambda_{j}[k]+d_{i} \cdot \delta \cdot\left(\lambda^{*}-\lambda_{i}[k]\right)
$$

The collective dynamics of a group of $n$ agents can be synthesized as follows:

$$
\boldsymbol{\lambda}[k+1]=\mathbf{A} \boldsymbol{\lambda}[k]+\operatorname{diag}\left(d_{i} \cdot \delta\right)\left(\lambda * \mathbf{1}_{n}-\boldsymbol{\lambda}[k]\right)=\mathbf{A}_{f} \boldsymbol{\lambda}[k]+\mathbf{A}_{f l} \lambda *
$$

where $\mathbf{A} \in \mathrm{R}^{\mathrm{n} \times \mathrm{n}}$ is the system information weighting matrix and its elements are defined by Formula (11). $\mathbf{A}_{f}=\mathbf{A}-\operatorname{diag}\left(d_{i} \cdot \delta\right)$, and $\mathbf{A}_{f l}=\delta\left[d_{1}, d_{2}, \cdots, d_{n}\right]^{\mathrm{T}}$ is called the virtual power generator identify vector. 
We define the system state error vector at the $k$-th iteration cycle as follows:

$$
z[k]=\lambda[k]-\lambda * \mathbf{1}_{n}
$$

The evolution of the system state error can be derived according to Formula (17) as follows:

$$
\begin{aligned}
z[k+1] & =\lambda[k+1]-\lambda * \mathbf{1}_{n} \\
& =\mathbf{A}_{f} \boldsymbol{\lambda}[k]+\mathbf{A}_{f l} \lambda *-\lambda * \mathbf{1}_{n} \\
& =\mathbf{A}_{f}\left(\lambda[k]-\lambda * \mathbf{1}_{n}\right)+\mathbf{A}_{f} \lambda * \mathbf{1}_{n}+\mathbf{A}_{f l} \lambda *-\lambda * \mathbf{1}_{n} \\
& =\mathbf{A}_{f} \boldsymbol{z}[k]
\end{aligned}
$$

The following lemma is needed [27] to analyze the convergence property of the system (17) [28]:

Lemma 1. If $\mathbf{A} \in \mathbf{M}^{m \times n}$, then $\rho(\mathbf{A})$ is an eigenvalue of $\mathbf{A}$ and there is a nonnegative vector $x \geq 0, x \neq 0$, such that $\mathbf{A} x=\rho(\mathbf{A}) x$.

Theorem 1. Consider a multi-agent system with the dynamic of (17) and with an undirected connected communication topology. If $\delta \in(0,1 /(d m a x+1))$, then the spectral radius of the matrix

$$
\mathbf{A}_{f}=\left[\begin{array}{cccc}
a_{11}-\delta & a_{12} & \cdots & a_{1 n} \\
a_{21} & a_{22} & \cdots & a_{2 n} \\
\vdots & \vdots & \ddots & \vdots \\
a_{n 1} & a_{n 2} & \cdots & a_{n n}
\end{array}\right]
$$

is less than 1 .

Proof. Let $v=\left[v_{1}, \ldots, v_{n}\right]^{\mathrm{T}}$ be the left eigenvector of $\mathbf{A}_{f}$ corresponding to the spectral eigenvalue $\rho\left(\mathbf{A}_{f}\right)$. Lemma 1 shows that $v \geq 0, v \neq 0$. Let $v_{q}=\max _{j=1,2, \ldots, n}\left(v_{j}\right)$. Thus, $v_{q}>0$.

If $q=1$, then

$$
\begin{aligned}
& \rho\left(\mathbf{A}_{f}\right) v_{1}=\sum_{j=1}^{n} a_{f, 1 j} v_{1}=-\varepsilon v_{1}+\sum_{j=1}^{n} a_{1 j} v_{j} \\
& \leq-\varepsilon v_{1}+\sum_{j=1}^{n} a_{1 j} v_{1}=-\varepsilon v_{1}+v_{1}<v_{1}
\end{aligned}
$$

That is to say: $\rho\left(\mathbf{A}_{f}\right)<1$ when $q=1$.

If $q \neq 1$, then

$$
\rho\left(\mathbf{A}_{f}\right) v_{q}=\sum_{j=1}^{n} a_{f, q j} v_{j}=\sum_{j=1}^{n} a_{q j} v_{j} \leq \sum_{j=1}^{n} a_{q j} v_{q}=v_{q}
$$

Thus, $\rho\left(\mathbf{A}_{f}\right) \leq 1$. However, if $\rho\left(\mathbf{A}_{f}\right)=1$, then $v=\left[v_{q}, . ., v_{q}\right]^{\mathrm{T}}$ as matrix $\mathbf{A}$ of a connected graph is irreducible. It is impossible since

$$
-\varepsilon v_{q}+v_{q}<v_{q}
$$

Thus, $\rho\left(\mathbf{A}_{f}\right)<1$.

By Theorem 1, we know that the spectral radius of $\mathbf{A}_{f}$ is less than 1. Thus, the system state error $z[k] \rightarrow 0$ as $k \rightarrow \infty$, which means that all the agents' state converge to the control target $\lambda^{*}$. 


\section{Simulation Verifications}

\subsection{Simulation Model}

We considered an MG integrated with five distributed generators and formed a six-agent system by counting the virtual generator. The parameter values of each distributed generator are referred to in reference [29], which are listed in Table 1. Figure 3 illustrates the communication network topology.

Table 1. Parameter values of each distributed generator.

\begin{tabular}{cccccc}
\hline Generator & $\left.\boldsymbol{a}_{\boldsymbol{i}} \mathbf{( \$ \mathbf { M W }} \mathbf{\mathbf { 2 }}\right)$ & $\boldsymbol{b}_{\boldsymbol{i}} \mathbf{( \$ \mathbf { M W } )}$ & $\boldsymbol{c}_{\boldsymbol{i}} \mathbf{( \$ )}$ & $\boldsymbol{P}_{\boldsymbol{G} \boldsymbol{i}}^{\min }(\mathbf{M W})$ & $\boldsymbol{P}_{\boldsymbol{G} \boldsymbol{i}}^{\max } \mathbf{( M W )}$ \\
\hline G1 & 0 & 0 & 0 & $-\infty$ & $+\infty$ \\
G2 & 0.0070 & 7.0 & 240 & 100 & 500 \\
G3 & 0.0095 & 10.0 & 200 & 50 & 200 \\
G4 & 0.0090 & 8.5 & 220 & 80 & 300 \\
G5 & 0.0080 & 11.0 & 200 & 50 & 150 \\
G6 & 0.0075 & 10.5 & 220 & 50 & 200 \\
\hline
\end{tabular}

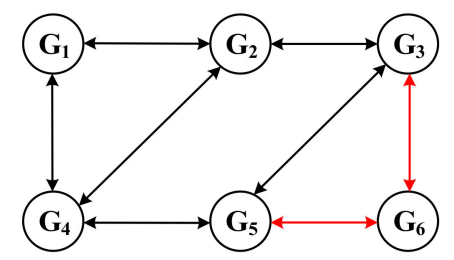

Figure 3. Topology of the communication network.

Simulation results under three scenarios are provided to study the performance of our real-time distributed ED strategy. In comparison with the distributed ED strategy in reference [30], the effectiveness and solution accuracy of the strategy are verified and analyzed in scenario 1 . Scenario 2 simulates the switch of the exchanged power order. The plug and play functionality of the strategy are demonstrated in scenario 3 .

\subsection{Scenario 1: Effectiveness Validation}

The total load demand of the MG is $1000 \mathrm{~kW}$ and power instruction $P_{\text {ref }}$ is $120 \mathrm{~kW}$. The initial power of all the generation units were set to the minimum of their output. The simulation was carried out in the case of neglecting and considering network power loss, respectively. Figure 4 shows the results of our distributed ED strategy with $\delta=0.003$.

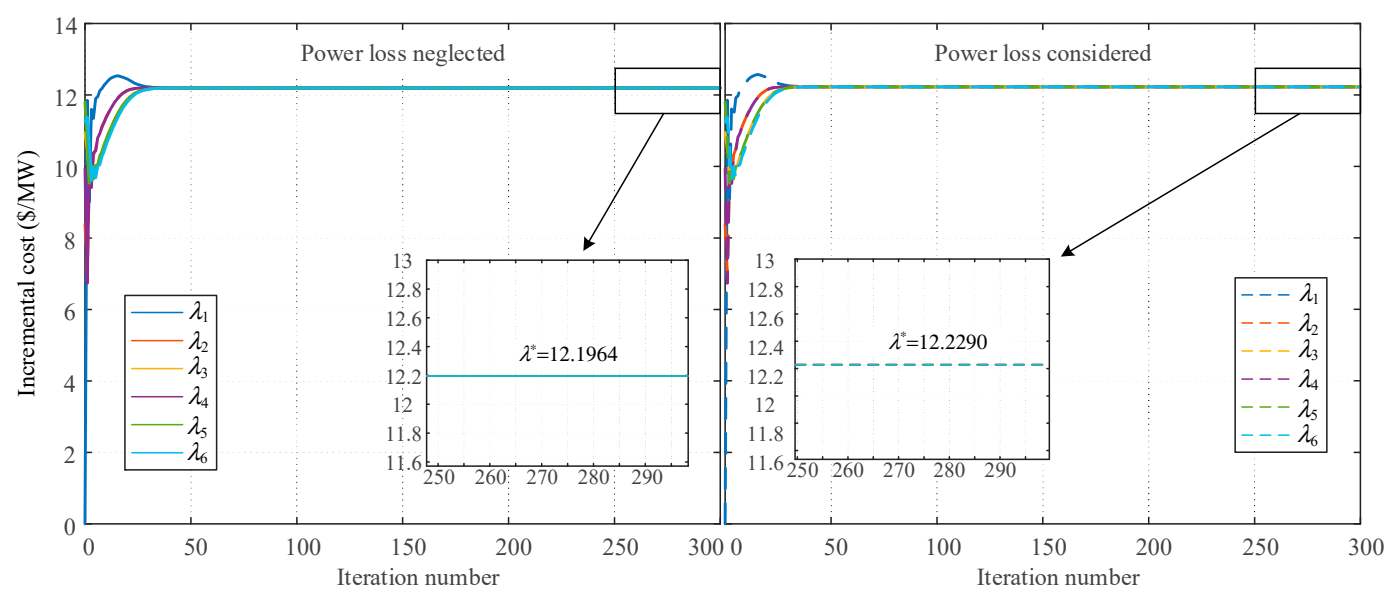

(a)

Figure 4. Cont. 


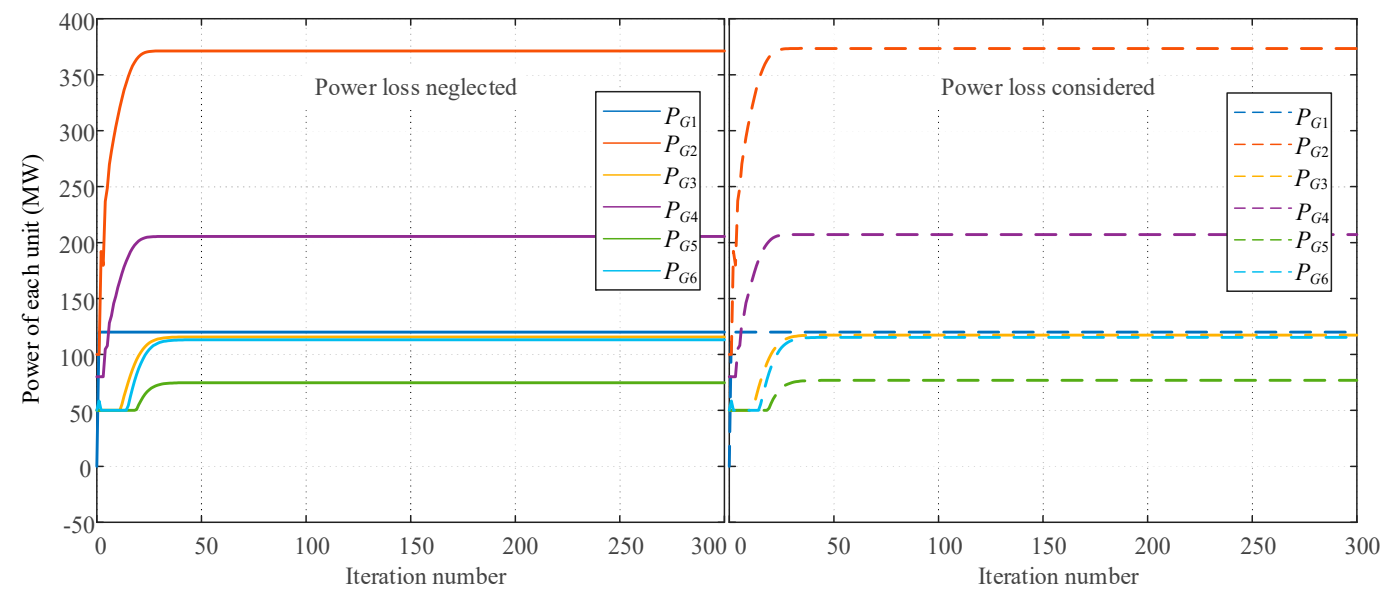

(b)

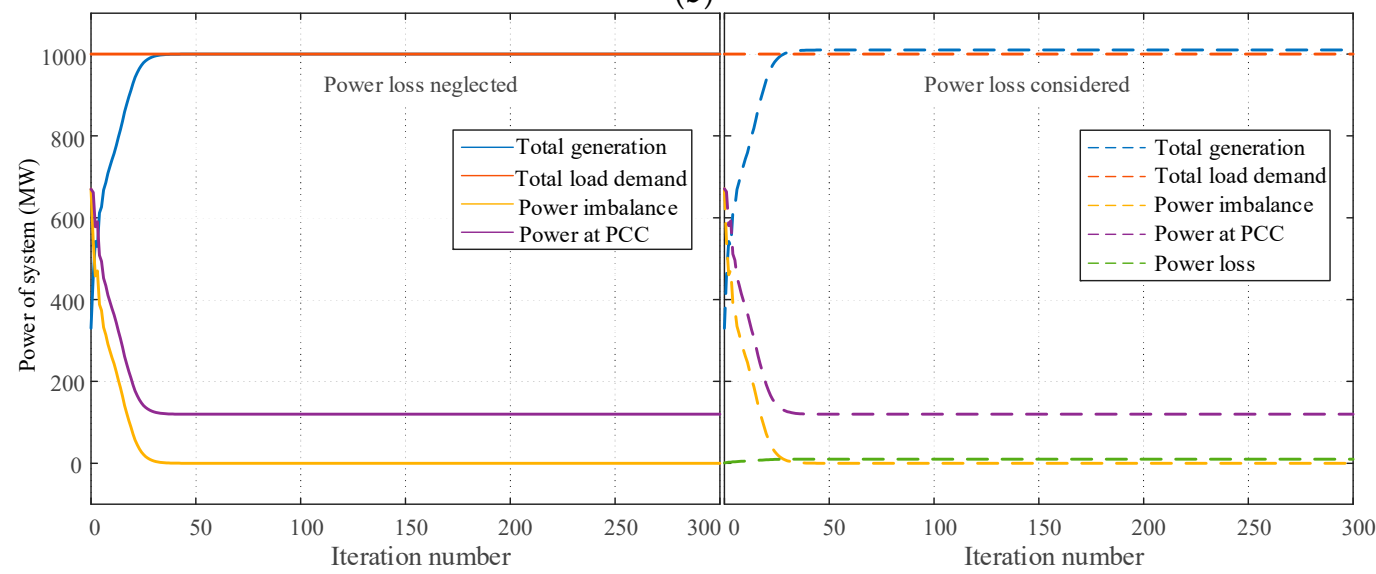

(c)

Figure 4. Simulation results of scenario 1. (a) incremental cost; (b) power of each unit; (c) power information of the system.

Using the distributed ED strategy in reference [30], the simulation results are shown in Figure 5. Note that, since the external grid was no longer regarded as a virtual generator, the total load of the system was set to $880 \mathrm{MW}$.

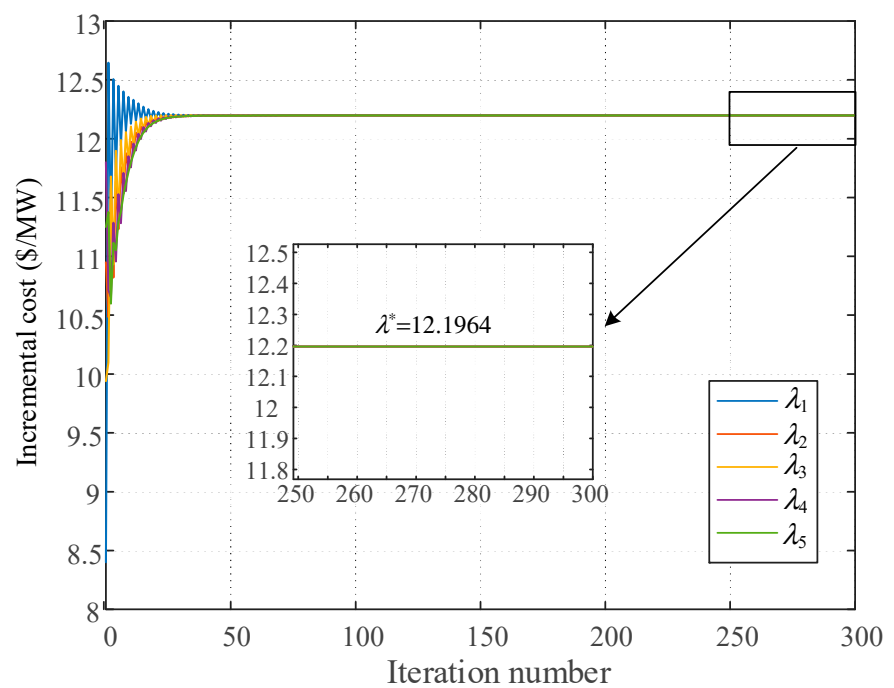

(a)

Figure 5. Cont. 


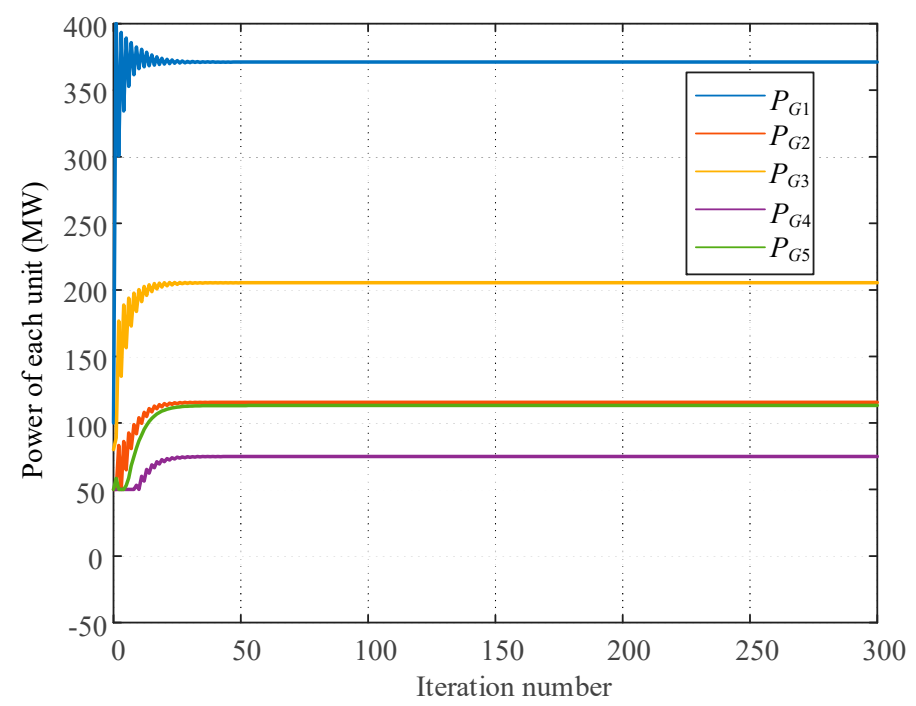

(b)

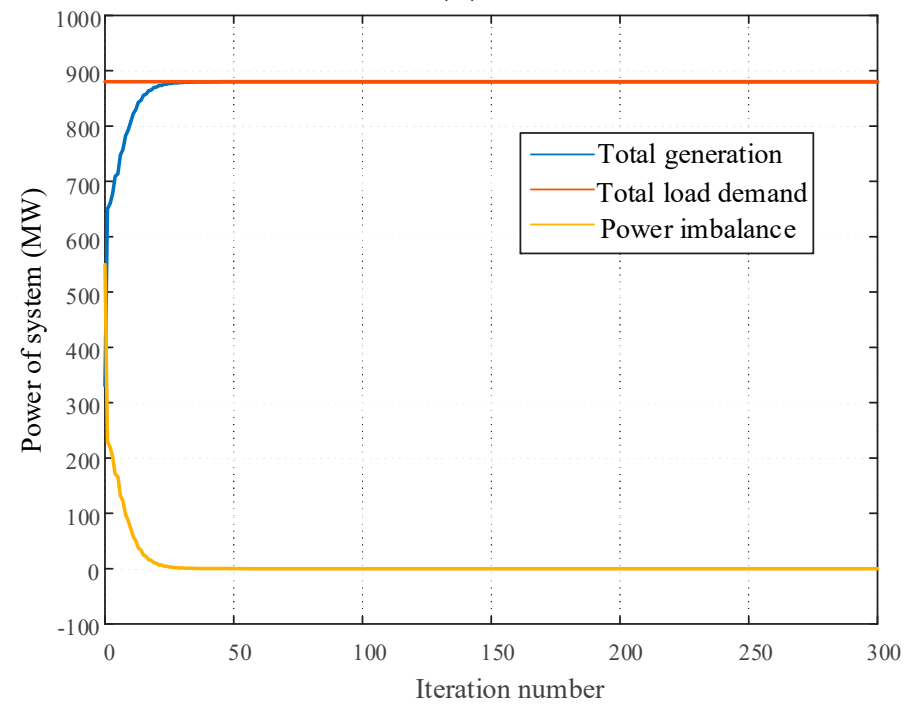

(c)

Figure 5. Simulation results by using the distributed ED strategy in reference [30]. (a) Incremental cost; (b) power of each unit; (c) power information of the system.

As shown in Figures 4 and 5, the distributed economic dispatch strategy adopted in this paper converges to the same value as the method in reference [30] without considering network loss. The incremental cost of all the power generation units eventually converge to $12.1964 \$ / \mathrm{MW}$, and the output power of the units in MG converge to $\boldsymbol{P}_{\mathrm{G}}{ }^{*}=[371.1725,115.6008,205.3564,74.7759,113.0943]$ (MW). Figure $4 \mathrm{c}$ shows that the exchanged power $P_{\mathrm{PCC}}$ finally converges to its instruction of $120 \mathrm{~kW}$, and the total power of all the generation units (including the $120 \mathrm{~kW}$ of the virtual generator) exactly balances the load demand $1000 \mathrm{~kW}$ of the MG. These results verify that our distributed strategy can solve the economic dispatch problem of an MG effectively and has the same solution accuracy as that of the method in [30].

However, we regarded the external power grid as a virtual generator that participates in power dispatch, meaning that the problem of global information being hard to obtain, as in reference [30], has been solved. At the same time, the setting of the $P_{\text {ref }}$ instruction makes the power regulation of the MG more flexible. More importantly, the method adopted in this paper can naturally take the power loss into account, as it can be considered as a variable load. Thus, the accuracy of the economic dispatch of an MG is improved. As we can ascertain from Figure 4, the final incremental cost is $\lambda^{*}=12.2290 \$ / \mathrm{MW}$ 
when the network power loss is incorporated, which is little larger than the $12.1964 \$$ /MW. Additionally, the final power allocation in the MG is $\boldsymbol{P}_{\mathbf{G}}{ }^{*}=[373.5005,117.3161,207.1670,76.8129,115.26709](\mathrm{MW})$. The difference of $10.0636 \mathrm{MW}$ between total power generation and total load demand is exactly the value of the power loss of the MG network. The difficulty of considering the network power loss in the economic dispatch process, which seems to be insuperable to existing distributed ED algorithms, now can be handled effectively by our strategy.

\subsection{Scenario 2: Power Order Change}

In this scenario, the exchanged power order $P_{\text {ref }}$ changed from $120 \mathrm{MW}$ to $-50 \mathrm{MW}$ at the instance of $k=150$, to simulate the variation of the energy trading mode of the MG. The simulation results are shown in Figure 6.

It can be ascertained from Figure 6 that at the instance of $k=150$, the MG switches from the electricity purchasing mode into the electricity selling mode. Accordingly, the incremental cost increases from $12.2290 \$ / M W$ to $12.7816 \$ / M W$ to answer this change, and the final power allocation in the MG is $P_{\mathrm{G}}{ }^{*}=[412.9700,146.3390,237.8656,111.3488,152.1054]$ (MW). The goal of selling a power of $50 \mathrm{MW}$ to the external power grid is also achieved. These results prove that our strategy also has a satisfying robustness in coping with the variation of the exchanged power order.

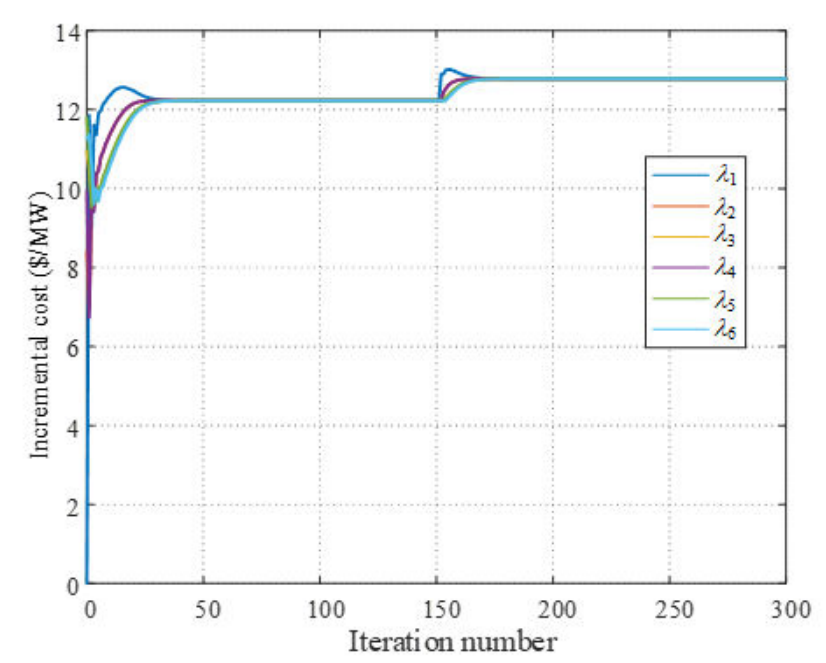

(a)

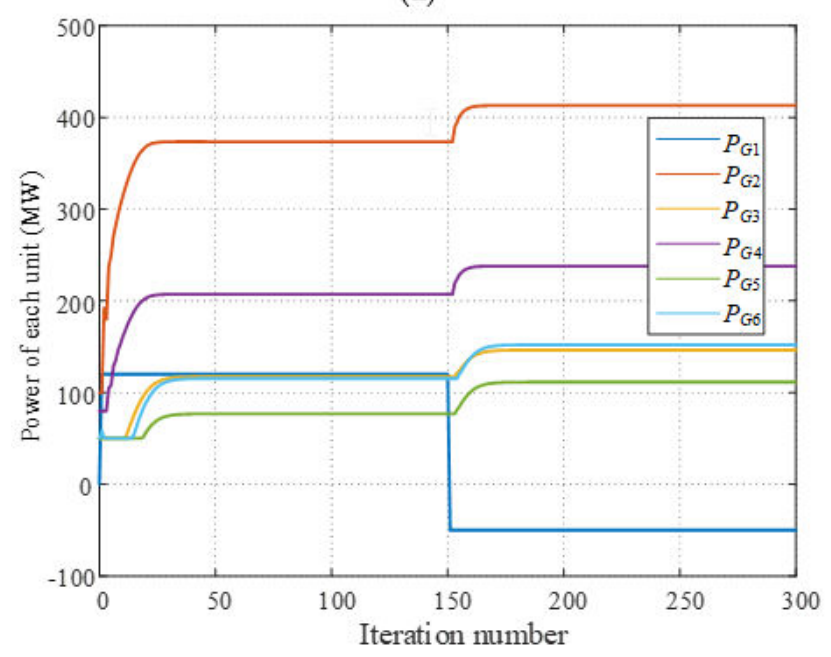

(b)

Figure 6. Cont. 


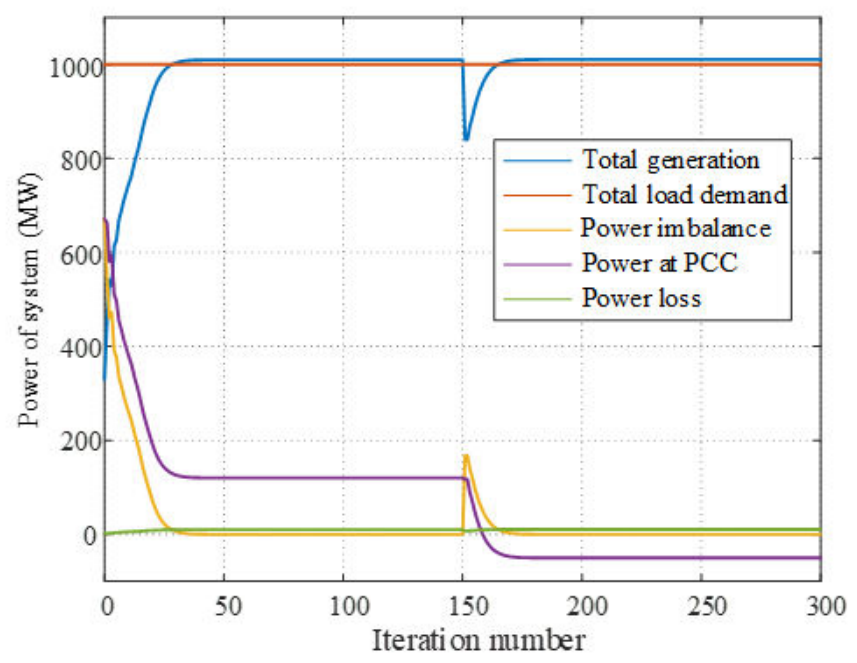

(c)

Figure 6. Simulation results of scenario 2. (a) Incremental cost; (b) power of each unit; (c) power information of the system.

\subsection{Scenario 3: Plug-and-Play Functionality Verification}

In this simulation, the power unit $G_{6}$ decided to disconnect from the MG at the instance of $k=$ 300 and to reconnect at $k=600$. The two communication links with a red color in Figure 3 are not available during the disconnection of $G_{6}$. Other settings are consistent with scenario 1 . Figure 7 shows the simulation results.

It can be seen from Figure 7 that at the instance of $k=300$, the remaining four power generation sources in the MG collaborate in order to cope with the islanding of $G_{6}$ by increasing their output power simultaneously. Their incremental costs increase from 12.2290 \$/MW to 12.7055 \$/MW to re-establish the balance between power generation and load demand. After the reconnection of $G_{6}$ at $k=600$, the incremental costs all converge back to the initial value of $12.2290 \$ / \mathrm{MW}$. This case study clearly shows that the proposed algorithm can provide a better plug-and-play capability for an MG, which is more appreciated in the situation of a high penetration level of distributed generations.

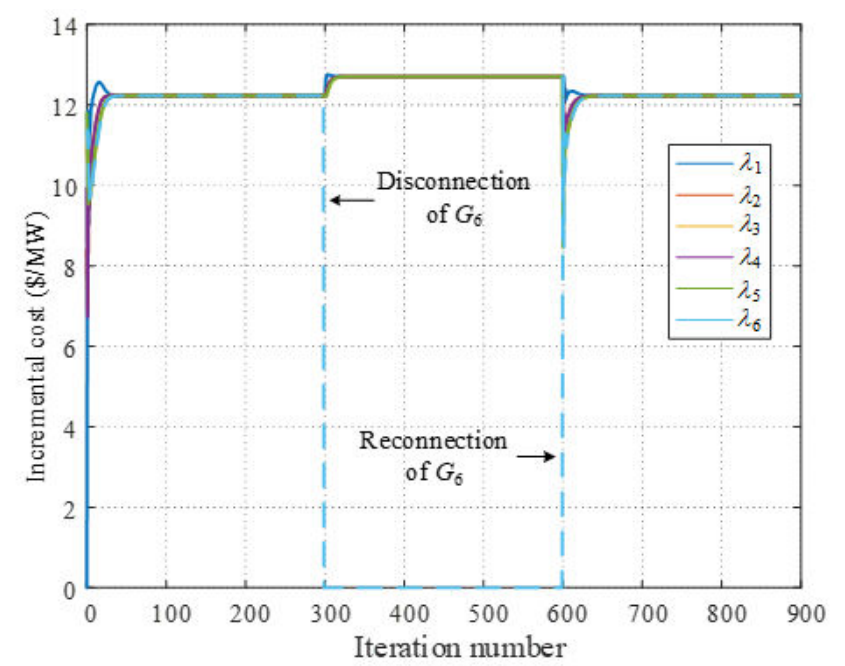

(a)

Figure 7. Cont. 


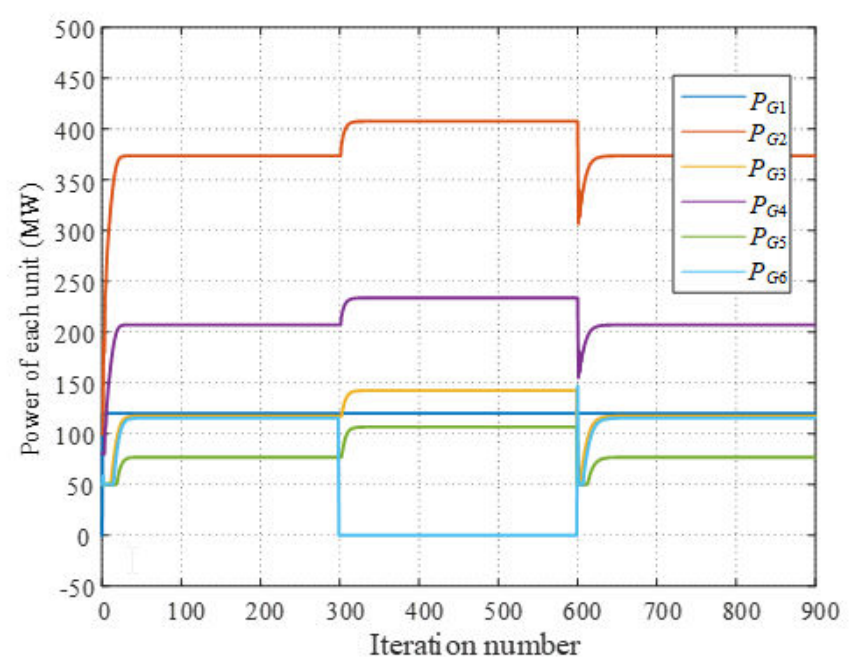

(b)

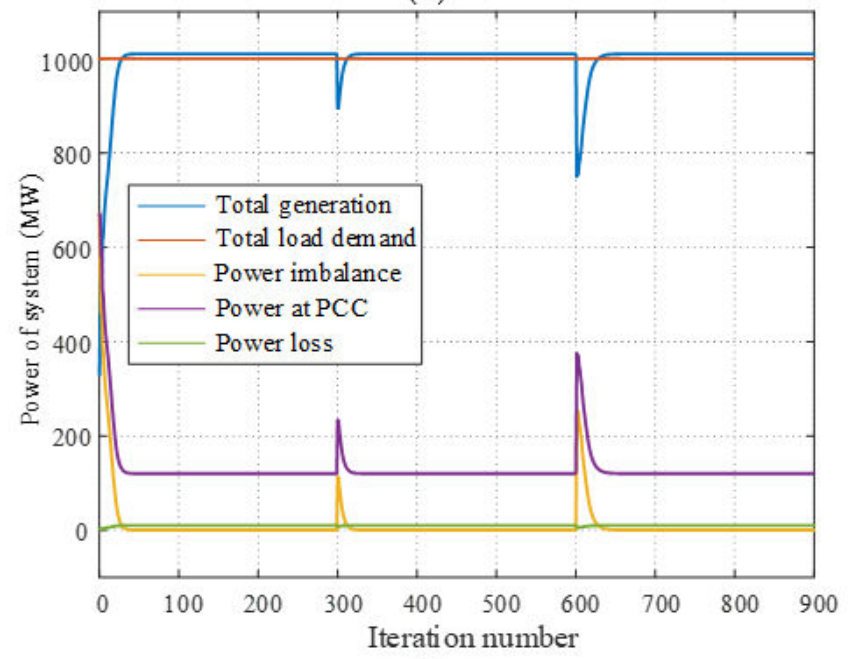

(c)

Figure 7. Simulation results of scenario 4. (a) Incremental cost; (b) power of each unit; (c) power information of the system.

\section{Conclusions}

Based on the principle of consensus theory and distributed sub-gradient optimization, this paper proposes a real-time strategy that can fulfill the task of the economic dispatch of MGs in a fully distributed fashion. The external power grid to which the MG connects is treated as a special power source called a virtual generator and participates in the ED process. Only the power exchanged between the external power grid and the MG should be measured and utilized locally by the virtual generator, and only the consensus variable, i.e., incremental cost, should be shared between power generation units and the virtual generator. In this way, a fully distributed dispatch system is achieved, and more importantly, troublesome issues such as the need of global information regarding the total load demand and network power loss can be tackled easily and completely.

Simulation results under several scenarios verify the effectiveness of the proposed strategy, and it has good robustness and stability for power volatility and exchanged power instruction variations, and the power unit plug-and-play. In comparison with the current distributed economic dispatch methods, the proposed strategy is a fully distributed version and is very simple to implement because it is insensitive to initial power settings and does not require any global information. In addition, it has a higher power dispatch accuracy due to the capability of network power loss handling. 
Author Contributions: Conceptualization, J.L. and Q.Z.; methodology, Q.Z.; validation, J.L., Q.Z. and L.Z.; formal analysis, Q.Z., and Y.W.; investigation, Q.Z.; data curation, Y.W.; writing-original draft preparation, Q.Z.; writing—review and editing, J.L., L.Z. and Y.W.; supervision, J.L.; funding acquisition, J.L.

Funding: This research received no external funding.

Conflicts of Interest: The authors declare no conflict of interest.

\section{References}

1. Pedrasa, M.A.A.; Spooner, T.D.; Macgill, I.F. Coordinated scheduling of residential distributed energy resources to optimize smart home energy services. IEEE Trans. Smart Grid 2010, 1, 134-143. [CrossRef]

2. Joo, I.Y.; Choi, D.H. Distributed optimization framework for energy management of multiple smart homes with distributed energy resources. IEEE Access 2017, 5, 15551-15560. [CrossRef]

3. Zandi, F.; Fani, B.; Sadeghkhani, I.; Orakzadeh, A. Adaptive complex virtual impedance control scheme for accurate reactive power sharing of inverter interfaced autonomous microgrids. IET Gener. Trans. Distrib. 2018, 12, 6021-6032. [CrossRef]

4. Zhao, Z.; Yang, P.; Guerrero, J.M.; Xu, Z.; Green, T.C. Multiple-time-scales hierarchical frequency stability control strategy of medium-voltage isolated Microgrid. IEEE Trans. Power Electron. 2016, 31, 5974-5991. [CrossRef]

5. Zhan, J.P.; Wu, Q.H.; Guo, C.X.; Zhou, X.X. Fast-iteration method for economic dispatch with prohibited operating zones. IEEE Trans. Power Syst. 2014, 29, 990-991. [CrossRef]

6. Kuo, M.T.; Lu, S.D.; Tsou, M.C. Considering carbon emissions in economic dispatch planning for isolated power systems-a case study of the Taiwan power system. IEEE Trans. Ind. Appl. 2017, 54, 987-997. [CrossRef]

7. Zhang, Y.; Hajiesmaili, M.H.; Chen, M. Peak-aware online economic dispatching for Microgrids. IEEE Trans. Smart Grid 2018, 9, 323-335. [CrossRef]

8. Li, P.; Xu, D.; Zhou, Z.; Lee, W.-J.; Zha, B. Stochastic optimal operation of Microgrid based on chaotic binary particle swarm optimization. IEEE Trans. Smart Grid 2017, 7, 66-73. [CrossRef]

9. Yuan, G.L.; Chen, S.L.; Liu, Y.; Fang, F. Economic optimal dispatch of virtual power plant based on time-of-use power price. Power Syst. Technol. 2016, 40, 826-832. [CrossRef]

10. Chen, G.; Li, C.; Dong, Z.Y. Parallel and distributed computation for dynamical economic dispatch. IEEE Trans. Smart Grid 2017, 8, 1026-1027. [CrossRef]

11. Chen, G.; Lewis, F.L.; Feng, E.N.; Song, Y. Distributed optimal active power control of multiple generation systems. IEEE Trans. Ind. Electron. 2015, 62, 7079-7090. [CrossRef]

12. Cady, S.T.; Domínguez-García, A.D.; Hadjicostis, C.N. A distributed generation control architecture for islanded AC Microgrids. IEEE Trans. Control Syst. Technol. 2015, 23, 1717-1735. [CrossRef]

13. Yun, Y.S.; Xia, Y.; Behdani, B.; Smith, J.C. Distributed algorithm for lifetime maximization in a delay-tolerant wireless sensor network with a mobile sink. IEEE Trans. Mob. Comput. 2013, 12, 1920-1930. [CrossRef]

14. Dong, X.W.; Yu, B.C.; Shi, Z.Y.; Zhong, Y. Time-varying formation control for unmanned aerial vehicles: Theories and applications. IEEE Trans. Control Syst. Technol. 2015, 23, 340-348. [CrossRef]

15. Mehrjerdi, H.; Saad, M.; Ghommam, J. Hierarchical fuzzy cooperative control and path following for a team of mobile robots. IEEE/ASME Trans. Mechatron. 2011, 16, 907-917. [CrossRef]

16. Tang, Z.; Hill, D.J.; Liu, T. A novel consensus-based economic dispatch for Microgrids. IEEE Trans. Smart Grid 2018, 9, 3920-3922. [CrossRef]

17. Chen, G.; Zhao, Z. Delay effects on consensus-based distributed economic dispatch algorithm in Microgrid. IEEE Trans. Power Syst. 2018, 33, 602-612. [CrossRef]

18. Yang, T.; Lu, J.; Wu, D.; Wu, J.; Shi, G.; Meng, Z.; Johansson, K.H. A distributed algorithm for economic dispatch over time-varying directed networks with delays. IEEE Trans. Ind. Electron. 2017, 64, 5095-5106. [CrossRef]

19. Zhang, Z.; Chow, M.Y. Convergence analysis of the incremental cost consensus algorithm under different communication network topologies in a smart grid. IEEE Trans. Power Syst. 2012, 27, 1761-1768. [CrossRef]

20. Park, M.J.; Kwon, O.M.; Ju, H.P.; Lee, S.-M.; Cha, E.-J. A new analysis on leader-following consensus for switched multi-agent systems with time-varying probabilistic self-delays. Int. J. Control Autom. Syst. 2015, 13, 611-619. [CrossRef] 
21. Binetti, G.; Abouheaf, M.; Lewis, F.; Naso, D.; Davoudi, A.; Turchiano, B. Distributed solution for the economic dispatch problem. In Proceedings of the 21st Mediterranean Conference on Control and Automation, Chania, Greece, 25-28 June 2013; pp. 243-250. [CrossRef]

22. Yang, S.; Tan, S.; Xu, J.X. Consensus based approach for economic dispatch problem in a smart grid. IEEE Trans. Power Syst. 2013, 28, 4416-4426. [CrossRef]

23. Guo, F.; Wen, C.; Mao, J.; Song, Y. Distributed economic dispatch for smart grids with random wind power. IEEE Trans. Smart Grid 2017, 7, 1572-1583. [CrossRef]

24. Binetti, G.; Davoudi, A.; Lewis, F.L.; Naso, D.; Turchiano, B. Distributed consensus-based economic dispatch with transmission losses. IEEE Trans. Power Syst. 2014, 29, 1711-1720. [CrossRef]

25. Liu, Z.F.; Liu, G.; Liu, X. Coordinated optimal dispatching of distributed generation based on quantum differential evolution algorithm. Power Syst. Technol. 2013, 37, 1922-1928. [CrossRef]

26. Nedic, A.; Ozdaglar, A. Distributed subgradient methods for multi-agent optimization. IEEE Trans. Autom. Control 2009, 54, 48-61. [CrossRef]

27. Horn, R.A.; Johnson, C.A. Matrix Analysis; Cambridge University Press: Cambridge, UK, 1985. [CrossRef]

28. Yang, W.; Wang, Y.; Wang, X.; Shi, H.; Ou, L. Optimal selection strategy for multi-agent system with single leader. In Proceedings of the 51st IEEE Conference on Decision and Control, Maui, HI, USA, 10-13 December 2012; pp. 2767-2772. [CrossRef]

29. Gaing, Z.L. Particle swarm optimization to solving the economic dispatch considering the generator constraints. IEEE Trans. Power Syst. 2003, 18, 1187-1195. [CrossRef]

30. Xie, J.; Chen, K.X.; Yue, D.; Li, Y.; Wang, K.; Wang, S.; Huang, C. Distributed economic dispatch based on consensus algorithm of multi agent system for power system. Electr. Power Autom. Equip. 2016, 36, 112-117. [CrossRef]

(C) 2019 by the authors. Licensee MDPI, Basel, Switzerland. This article is an open access article distributed under the terms and conditions of the Creative Commons Attribution (CC BY) license (http://creativecommons.org/licenses/by/4.0/). 